\title{
Trauma Abdominal
}

\author{
Abdominal Trauma
}

\author{
David A. Ortega-Checa ${ }^{1}$, Iván Vojvodic-Hernández ${ }^{2}$
}

\section{Introducción}

El trauma es el daño mecánico en la anatomía humana causada por una fuerza externa. También se define como el daño tisular por una energía dañina: la energía cinética es la más frecuentemente comprometida, pero también puede ser calórica, química o radioactiva. De la misma manera, puede producirse por la ausencia de un elemento esencial para la vida como el oxígeno que causa hipoxia y el calor que causa hipotermia $^{(1)}$.

El trauma es un verdadero problema de salud pública que tiene implicancias humanas y económicas, en países desarrollados es la primera causa de muerte antes de los 44 años y deja secuelas en un porcentaje importante en los pacientes que logran recuperarse de la lesión. Económicamente representa una gran pérdida, no solo monetaria sino de horas de dedicación al cuidado de estos pacientes ${ }^{(2)}$.

Los diferentes mecanismos de trauma son tan numerosos como las lesiones que causan en los pacientes. Por lo que fue necesario crear pautas que unifiquen criterios de diagnóstico y tratamiento para establecer un pronóstico de complicaciones y sobrevida. Es así que nacen los índices de severidad del trauma, que ayudan a los profesionales que manejan el taruma a "hablar el mismo idioma" (3).

Para estandarizar el diagnóstico y pronóstico del trauma se han diseñado diversos índices. Los índices existentes se clasifican en fisiológicos, anatómicos y mixtos. Entre los fisiológicos, esta la Escala de Coma de Glasgow, el Índice de Trauma Revisado, APACHE, etc., todos ellos se basan en los cambios fisiológicos que ocurren en el organismo como respuesta al trauma ${ }^{(3)}$.

Los índices anatómicos fueron creados para comparar y evaluar la severidad de la lesión categorizando el daño anatómico, a diferencia de los índices fisiológicos, según la evaluación de la magnitud de éste. Entre estos tenemos el ICD-
10, el Índice de Trauma Abdominal, Índice de Severidad de la Lesión $^{(3)}$.

En 1990 la AAST presentó la Escala de Lesiones de Órganos (Injury Scoring Scala) donde asigna grados a las lesiones de cada órgano abdominal del 1 al 5, de menor a mayor severidad. Es actualmente la más utilizada ${ }^{(4)}$.

Estos sistemas de puntuación están lejos de ser una ciencia exacta, pero son de gran utilidad para valorar el manejo de pacientes, los diferentes algorritmos, la efectividad de los resultados y de los sistemas. También tienen gran importancia en la investigación y control de calidad. Todos estos índices tienen fortalezas y debilidades por lo que debemos estar familiarizados con ellos para leer e interpretar críticamente la literatura $^{(5)}$.

El trauma en general es consecuencia, en el mayor porcentaje de casos, de accidentes de tránsito. La complejidad de la presentación esta asociada con las medidas de seguridad de la industria automotriz en la fabricación de los automóviles. "Los efectos combinados de control de velocidad, el uso mandatorio de cinturones de seguridad, bolsas de aire y tecnología avanzada en el frenado de vehículos han contribuido a una redistribución del tipo de lesiones observadas en trauma abdominal; con menor incidencia de lesiones cerebrales pero con un incremento de lesiones torácicas y abdominales"(6).

El TEC es la causa principal de muerte en trauma originado por accidentes de tránsito, siguen el trauma torácico (25\%) y el trauma abdominal $(13-15 \%)^{(7,8)}$.

En los Estados Unidos, anualmente las lesiones representan casi 200,000 muertes y más de 30 millones de lesiones no fatales ${ }^{(9)}$.

En el Perú, según estadísticas desde el año 2012, se registra un elevado número de accidentes de tránsito que producen heridos y víctimas fatales. El incremento es llamativo

${ }^{1}$ Ex-Jefe del Departamento de Cirugía General y Digestiva del Hospital Nac. Edgardo Rebagliati - EsSalud. Profesor Universitario . Ex Presidente de la Sociedad Panamericana de Trauma. Director del Curso ATLS - Perú. Código ORCID: 0000-0002-5448-3736. ${ }^{2}$ Profesor Principal de la Universidad de San Martin de Porres. Ex-Jefe del Departamento de Cirugía General y Digestiva del Hospital Nac. Edgardo Rebagliati - EsSalud. Código ORCID 0000-0002-4822-480X. 
en el año 2018 donde el promedio fue de 1,358 heridos y de 9 muertos por día ${ }^{(10)}$.

\begin{tabular}{|cccc|}
\multicolumn{5}{c|}{ Tabla 1 } \\
\hline \multicolumn{4}{c|}{$\begin{array}{c}\text { Estadísticas de accidentes de tránsito } \\
\text { que producen heridos y víctimas fatales }\end{array}$} \\
\hline Año & Accidentes de tránsito & Heridos & Muertos \\
\hline 2012 & 94,923 & 54,572 & 3,313 \\
2013 & 102,762 & 59,453 & 3,110 \\
2014 & 101,104 & 58,148 & 2,788 \\
2015 & 95,532 & 56,499 & 2,965 \\
2016 & 89,304 & 53,776 & 2,696 \\
2017 & 88,168 & 64,970 & 28,25 \\
2018 & 90,056 & 81,512 & 3,244 \\
\hline
\end{tabular}

El abordaje del paciente con trauma abdominal tiene como objetivo identificar y tratar las lesiones que amenacen la vida en forma inmediata, para lo cual el paciente debe ser evaluado siguiendo el protocolo de ATLS $^{(2)}$.

\section{Clasificación}

El trauma abdominal se divide en dos grandes grupos, de acuerdo a su mecanismo de producción: cerrado o contuso y abierto o penetrante. Ambos son una importante fuente de morbimortalidad, por lo que el diagnóstico precoz resulta de gran importancia para disminuir esta letalidad.

Hay que tener en cuenta que estas lesiones generalmente son multisistémicas, es decir pueden comprometer varios órganos y/o regiones, no es infrecuente que un paciente presente TEC asociado a lesión abdominal, cobra mayor importancia esta asociación pues el examen clínico del abdomen es poco confiable. Lo mismo ocurre con intoxicación alcohólica o lesión de la medula espinal. Esto obliga a utilizar métodos diagnósticos como el Lavado Peritoneal Diagnóstico, Ecografía, Tomografía o Laparoscopía.

En el trauma abdominal cerrado, que están asociadas a accidentes de tránsito, caídas de altura y contusiones con armas romas, los órganos mas afectados son el hígado y el bazo y las lesiones que se producen son:

1.Aplastamiento directo de órganos entre la pared abdominal anterior y posterior.

2.Lesiones por avulsión por fuerzas de desaceleración, como sucede en accidentes de alta velocidad o caídas desde alturas.

3. Formación transitoria de un asa intestinal cerrada con presión intraluminal alta y ruptura de la víscera hueca.
4. Laceraciones por fragmentos óseos (Ej., Pelvis, parte inferior costillas).

5. Lesiones del diafragma o incluso ruptura cardíaca ${ }^{(11)}$.

Existe mayor posibilidad de lesión abdominal cuando hay signos de trauma producido por el cinturón de seguridad del automóvil, como son: abrasión hematoma de pared o fractura de columna lumbar.

En el trauma penetrante el órgano más frecuentemente comprometido, en nuestro medio, es el intestino delgado e hígado ${ }^{(12)}$. Además el trauma penetrante se divide en cortopunzantes (arma blanca) y por proyectiles de arma de fuego (PAF). En el trauma penetrante siempre hay una solución de continuidad en pared abdominal y las lesiones que causan son menos graves que en el trauma cerrado, salvo que comprometa vasos sanguíneos importantes ${ }^{(7)}$

Las causas más frecuentes son las producidas por PAF y los órganos más frecuentemente comprometidos son intestino delgado, hígado, estómago, colon y estructuras vasculares. $(7,8,13)$.

\section{Evaluación Clínica}

La anamnesis, cuando se pueda realizar, nos permite conocer el probable mecanismo de producción y orienta al clínico sobre las probables lesiones que pueden presentarse.

En el examen físico es de capital importancia establecer los signos vitales. La presión arterial sistólica mayor de $90 \mathrm{~mm}$ Hg y una frecuencia cardíaca menor de 100/min, catalogan al paciente como hemodinámicamente estable y permite una mejor evaluación física. En ausencia de lesión craneal y espinal, los signos clínicos que podemos encontrar son: dolor abdominal, resistencia e hipersensibilidad, reacción peritoneal, que implican indicación quirúrgica. La ausencia de matidez hepática y de RHA son otros signos que pueden hacer sospechar la presencia de compromiso intraperitoneal. Igualmente la percusión dolorosa del abdomen puede indicar irritación peritoneal. Se debe tener presente que un paciente con hipovolemia y sin lesión evidente de hemotórax, ni de pelvis ni de huesos largos, la única cavidad que puede albergar una gran hemorragia es la cavidad abdominal ${ }^{(2,13,7,14)}$.

Nunca debe dejar de realizarse el examen rectal y de la pelvis. En el primer caso, el tacto rectal es importante para descartar sangrado, dolor a ese nivel e hipotonicidad del esfinter y la palpación adecuada de la próstata para descartar probable lesión de uretra posterior. La pelvis debe ser evaluada 
para descartar lesiones, se debe establecer la estabilidad a través de la ausencia de dolor (se debe presionar con delicadeza las crestas iliacas y la sínfisis) pues puede producirse fracturas que causan una gran hemorragia que puede ser causa de muerte (15).

El examen abdominal debe ser repetido, de ser posible por un mismo examinador tantas veces como sea necesario, buscando variaciones y signos para determinar un diagnóstico temprano.

Finalmente no olvidar desnudar totalmente al paciente y examinar la parte lateral y posterior del abdomen, así como la región perineal $^{(6,8)}$.

\section{Métodos Diagnósticos}

\section{Radiografía de Tórax y Abdomen}

La radiografía de Tórax anteroposterior puede dar importante información cuando se presenta un trauma de esta región, hay que descartar: neumotórax, desviación de la tráquea, ensanchamiento de mediastino, aumento de la silueta cardíaca, lesiones de costillas y sospecha de ruptura diafragmática..En la incidencia de pie se puede determinar presencia de neumoperitoneo ${ }^{(2)}$.

La radiografía simple de abdomen puede demostrar neumpoperitoneo, hay que descartar fracturas de costillas, pelvis y cuerpos vertebrales ya estas lesiones se asocian a compromiso visceral intraperitoneal. Se necesitan al menos $800 \mathrm{ml}$. de sangre intraperitoneal para ser evidente en una radiografía simple de abdomen. Según las condiciones del paciente no siempre es posible realizar este examen en posición de pie, lo cual es una limitación ${ }^{(16)}$.

Se recomienda que la radiografía de abdomen involucre la pelvis para evaluar la presencia de fracturas ${ }^{(2)}$.

\section{Lavado Peritoneal Diagnóstico}

Es de los procedimientos más antiguo, e igual que la radiografía simple de abdomen son probablemente los que menos se utiliza en la actualidad. El LPD continúa siendo un procedimiento de gran utilidad y de fácil realización. Puede ser abierto o cerrado (percutáneo), siendo el más usado el método abierto. Tiene una alta sensibilidad pero baja especificidad. No es útil en lesiones retroperitoneales y de diafragma. Está indicado cuando no esta disponible la ecografía o la TAC y en todo paciente con hipotensión en el que no se demuestra lesión torácica, pélvica o lesiones de huesos largos y que presente algunas de las siguientes condiciones:

-Cambios en el estado de conciencia-lesión cerebral, intoxicación alcohólica y uso de drogas ilícitas

- Cambios de la sensibilidad por probable lesión medular

-Lesiones de estructuras adyacentes: parrilla costal inferior, pelvis, columna lumbar

-Examen abdominal dudoso

-Pacientes con indicación quirúrgica extraabdominal

La única contraindicación absoluta es cuando se ha determinado una Laparotomía exploradora.

Se considera LPD positivo:

-Aspiración de mas de 10 cc de sangre macroscópica

-Globulos rojos $+100,000 / \mathrm{ml}$

-Leucocitos $+500 / \mathrm{ml}$

-Amilasa + $175 \mathrm{UI} / \mathrm{dl}$

-Presencia de bilis, bacterias o residuos alimentarios

Un LPD positivo en un paciente en inestabilidad hemodinmica debe considerarse como sinónimo de sangrado intrabadominal por lo que se indica una exploración quirúrgica abdominal $^{(17)}$.

En caso de gestación o trauma pélvico, el LPD debe realizarse por encima del ombligo ${ }^{(2,8,7)}$. (Tabla 2).

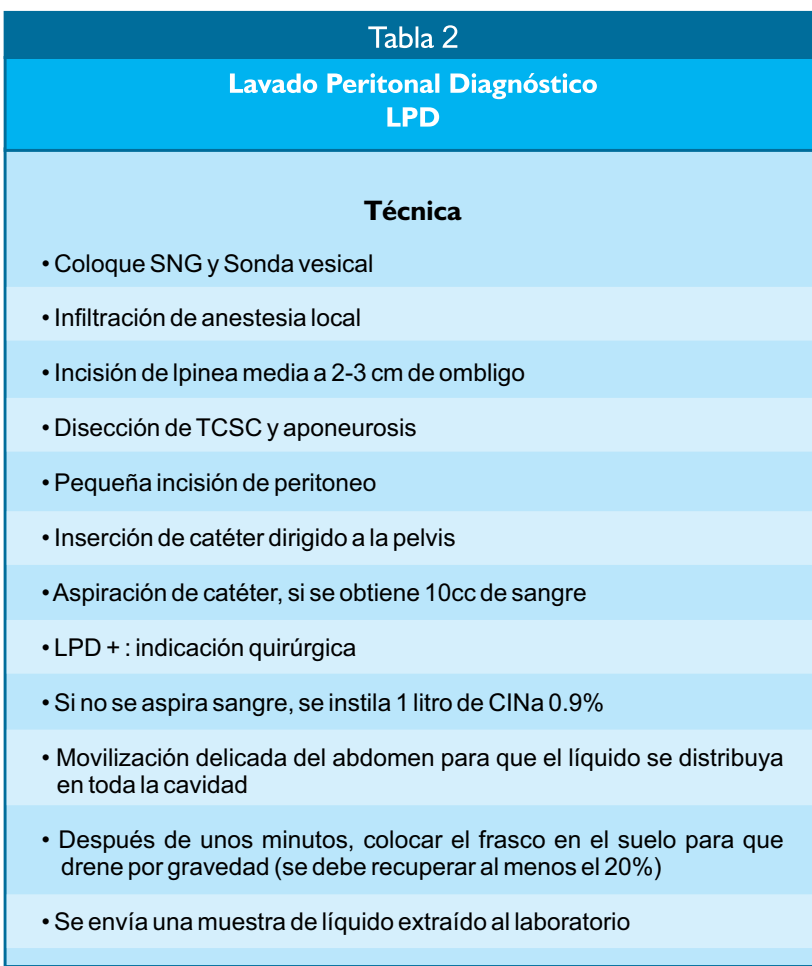




\section{Ecografía}

Actualmente es el método más utilizado, ha desplazado al LPD. Tiene la ventaja de no ser invasivo, se puede repetir cuanta veces se crea necesario, es de bajo costo, rápido, se puede realizar con equipo portátil en la sala de examen. Tiene un $90 \%$ de sensibilidad y $100 \%$ de especificidad ${ }^{(7)}$.

Tiene como objetivo detectar líquidos en la cavidad peritoneal, que en el contexto del trauma abdominal se interpreta como sangre, sin embargo no puede diferenciar el hemoperitoneo de otros tipos de fluidos (por ejemplo ascitis o secreción intestinal). Otra desventaja es la dificultad que presenta en pacientes con marcada distensión abdominal, dado que el aire impide una adecuada exploración de la cavidad abdominal, también cuando hay enfisema subcutáneo la exploración se dificulta. Debe realizarse con médicos preparados y se va a depender de su experiencia ${ }^{(17)}$.

\section{Las indicaciones son las mismas del LPD}

La ecografía abdominal en la evaluación del trauma utiliza una técnica específica que se denomina Ecofast o FAST que explora cuatro "ventanas ecográficas":

1.Región subcostal derecha, se busca localizar el espacio de Morrinson.

2.Epigastrio, región subxifoidea, se visualiza el saco pericárdico para descartar hemopericardio.

3. Región subcostal izquierda, se descarta liquido en celda esplenorrenal.

4. Región suprapúbica, se localiza fondo de saco de Douglas y espacio retrovesical.

Un paciente con trauma abdominal e hipotensión y una ecografía abdominal positiva tiene indicación quirúrgica. ${ }^{(6,7,7,15,18)}$. (Figura 1A-1B).

\section{Tomografía Computada TAC}

Es un procedimiento de gran utilidad pues brinda información sobre los órganos sólidos y en caso de lesión de alguno de éstos se puede evaluar la extensión de la misma, de igual manera puede indicar la presencia de líquidos y de aire intra abdominal. Además permite evaluar toda la cavidad peritoneal, la pelvis y el espacio retroperitoneal. Tiene la desventaja que el paciente debe estar hemodinámicamente estable, debe ser trasladado al tomógrafo y se debe utilizar

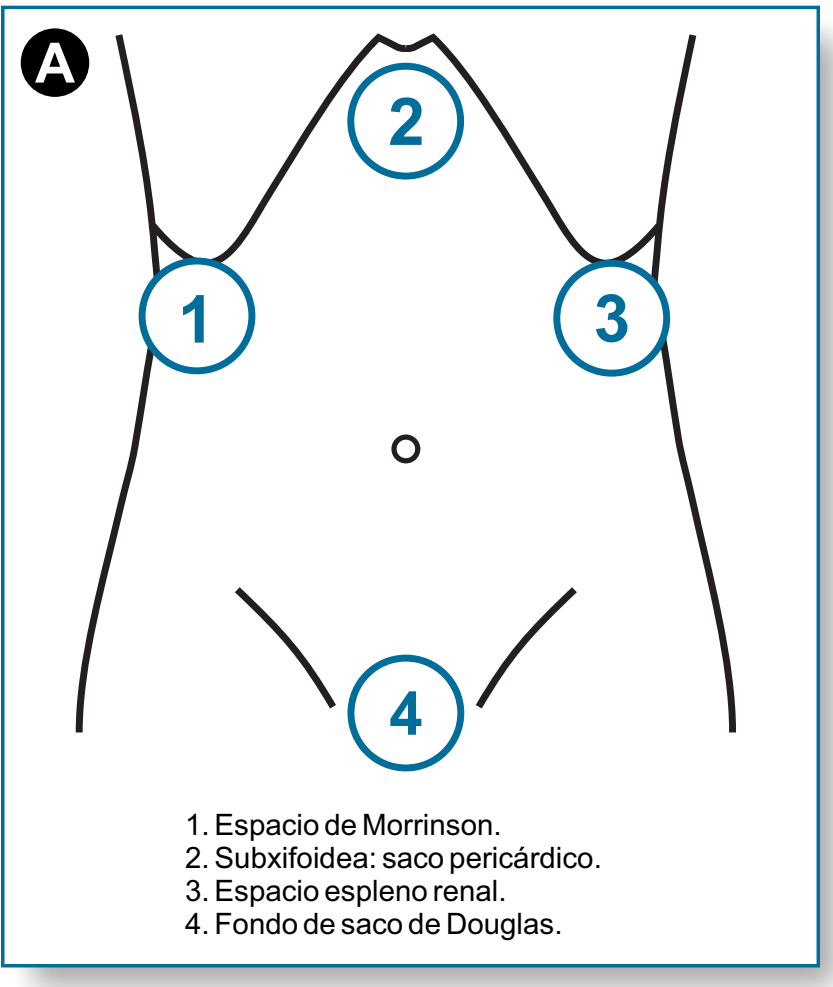

Figura 1A. Ecografía abdominal. Ventanas ecográficas.

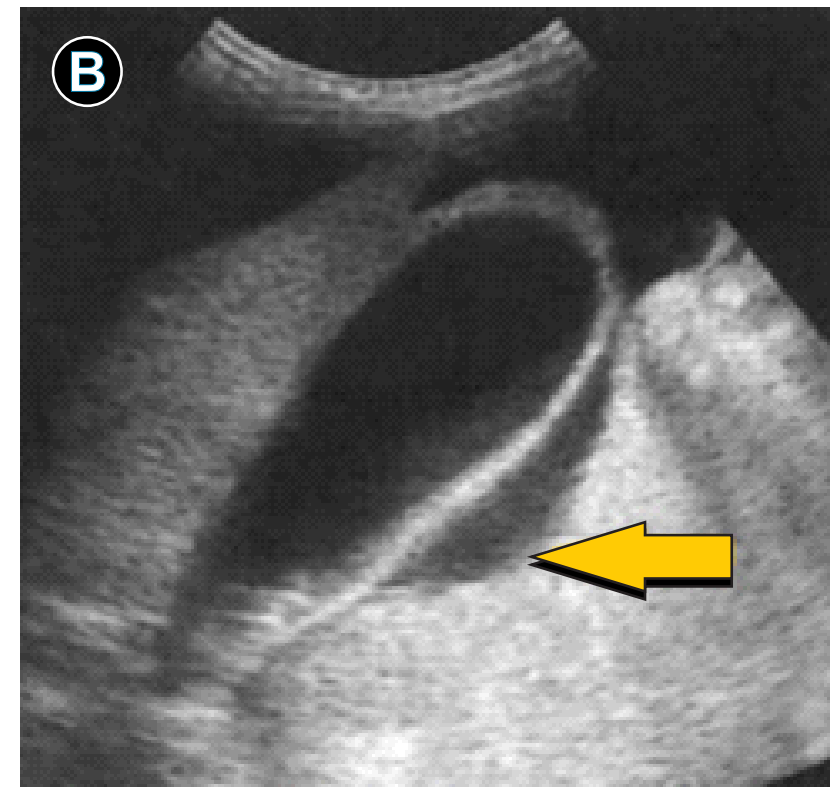

Figura 1 B. Ecografía : líquido libre en cavidad.

sustancia de contraste endovenoso. Igualmente es costoso y sobretodo consume tiempo. Algunas lesiones gastrointestinales, diafragmáticas y pancreáticas pueden pasar desapercibidas. Tiene una alta sensibilidad (92-98\%) para el diagnóstico de lesiones en trauma abdominal cerrado ${ }^{(2)}$. 
El desarrollo de la tecnología ha permitido una nueva generación de tomógrafos que permite una evaluación más rápida y con una mejor resolución con lo que brinda mejores imágenes de las lesiones tanto de órganos sólidos, de las vísceras y de los vasos sanguíneos ${ }^{(15,7,74)}$. (Figura 2). existe perforación del peritoneo. En los últimos años ha aumentado su utilización tanto para el diagnóstico como tratamiento. Se trata de identificar las lesiones y si es posible realizar el tratamiento con este abordaje $\mathrm{e}^{(20-21)}$.

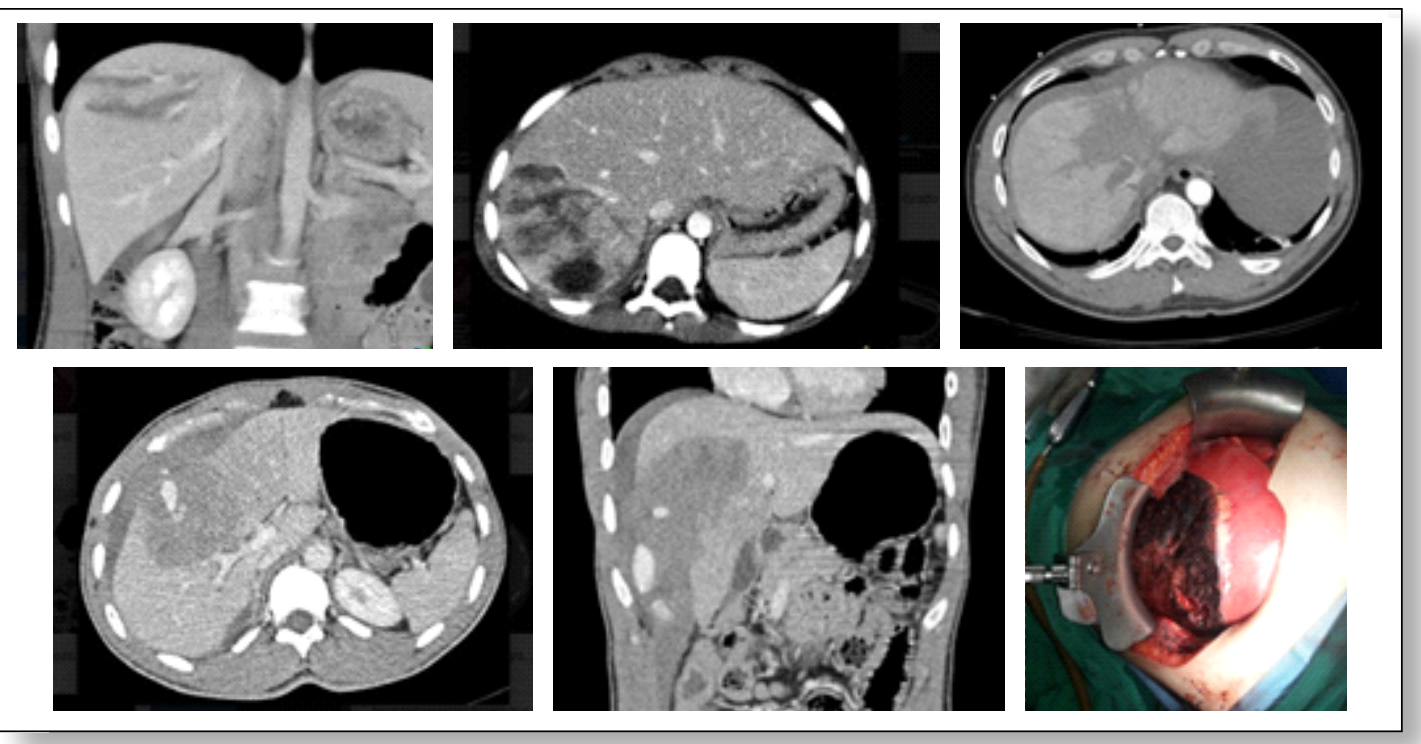

Figura 2. Tomografías computadas que muestran lesiones hepáticas en diferentes grados y su correlación con el hallazgo operatorio en las imágenes inferiores.

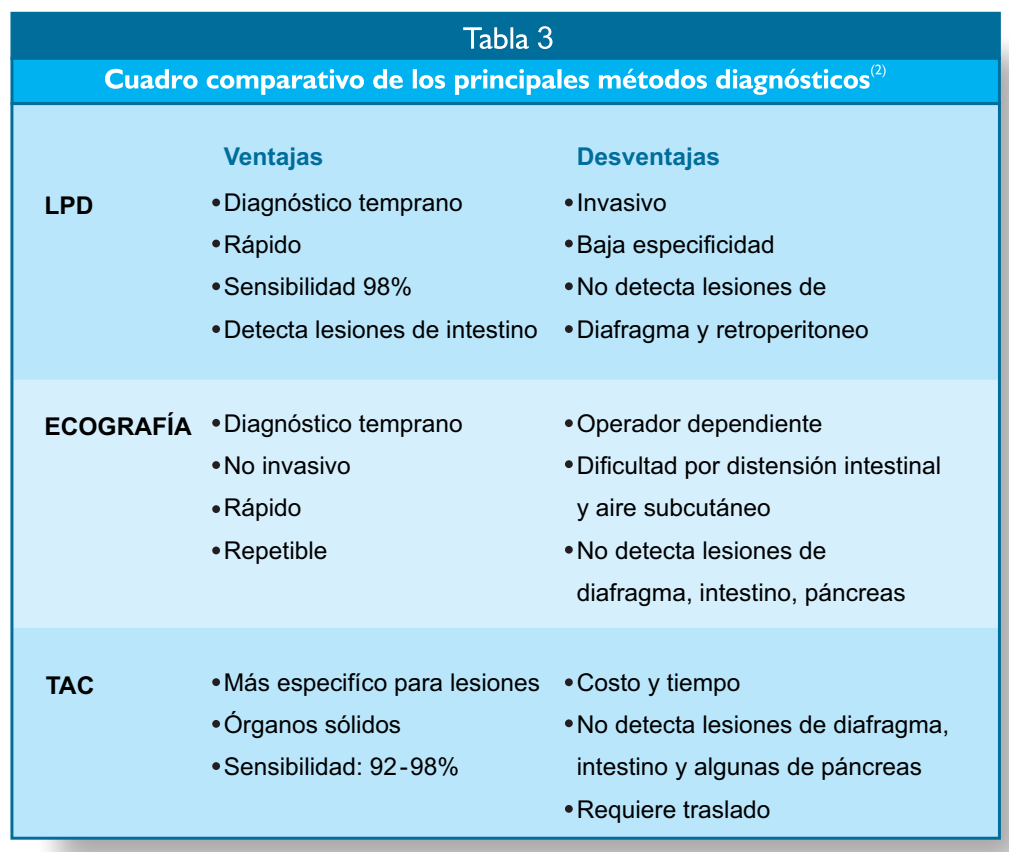

\section{Laparoscopía}

Inicialmente descrita en el manejo del trauma penetrante en heridas tangenciales por PAF para determinar si
Está indicado en paciente hemodinámicamente estables cuando los otros procedimientos de diagnóstico son dudosos de acuerdo al cuadro clínico. Es muy útil para descartar lesión de diafragma, pero no muy confiable pues es difícil realizar una 
adecuada exploración de toda la cavidad peritoneal, sin embargo se obtienen mejores resultados de acuerdo a la experiencia del cirujano ${ }^{(21)}$.

La laparoscopía adquiere gran importancia en pacientes con trauma penetrante de la región toracoabdominal pues tiene la capacidad de confirmar o descartar la penetración peritoneal para la toma de decisiones y además va a determinar la cavidad a explorar: tórax o abdomen ${ }^{(6,15,21,22)}$.

Las contraindicaciones son: indicación de laparotomía exploradora, signos de peritonitis, inestabilidad hemodinámica y TEC grave (la insuflación aumenta la presión intracraneal) $)^{(7)}$.

\section{Exploración de la herida en trauma penetrante}

En paciente con herida penetrante por arma blanca y en ausencia de inflamación peritoneal e inestabilidad hemodinámica es muy útil explorar la herida para estimar la profundidad de la penetración del arma en la pared abdominal para determinar si hubo ingreso a la cavidad peritoneal.

Existe la llamada regla de los tres tercios, un tercio no llega a penetrar el peritoneo, un tercio penetra el peritoneo pero no causa lesiones intraperitoneales, y otro tercio, penetra el peritoneo con lesiones intracavitarias ${ }^{(6)}$.

La exploración puede realizarse en la sala de examen, se utiliza anestesia local, si es necesario se puede ampliar la herida y se realiza una exploración digital o instrumental. Se considera positiva cuando la herida penetra a la cavidad ${ }^{(15,8)}$.

\section{Manejo e Indicación Quirúrgica}

Depende del tipo de trauma abdominal, cerrado o abierto. Sin embargo, al margen del tipo la condición más importante es establecer si el paciente es hemodinámicamente estable. En base a ello se determinará los pasos a seguir. En el caso de mantenerse la inestabilidad hemodinámica pese a las medidas de reanimación, el paciente deberá ser intervenido quirúrgicamente sin excepción.

\section{Trauma cerrado:}

En todo paciente, con o sin inestabilidad hemodinámica, se procede a realizar la reanimación siguiendo el protocolo $\operatorname{ABCDE}(\mathrm{ATLS})^{(2)}$.

Tiene indicación quirúrgica mandatoria los pacientes hemodinámicamente estables con presencia de irritación peritoneal. Si la presencia de peritonitis es negativa o dudosa se procede a realizar una Ecografía o TAC o LPD, de acuerdo a la disponibilidad en el servicio de emergencia, la mayoría prefiere la ecografía por su rapidez y bajo costo, de no contar con este examen se debe realizar LPD. Si el examen es positivo en detectar líquido libre en cavidad por la ecografía o sangre por el LPD se indica exploración quirúrgica. En caso de ser negativo se procede a realizar observación y reevaluación. Se debe tener presente que los casos de traumatismo hepático grado I y II (los que se caracterizan por ser lesiones de menos de $3 \mathrm{~cm}$ de profundidad y menos de $10 \mathrm{~cm} \mathrm{de)} \mathrm{los} \mathrm{pacientes} \mathrm{son} \mathrm{tributarios}$ de manejo no operatorio a pesar de ser positivo a la Ecografía o LPD siempre y cuando el paciente curse hemodinámicamente estable y la institución disponga de TAC para el seguimiento. ${ }^{(2,7)}$. (Figura 3).

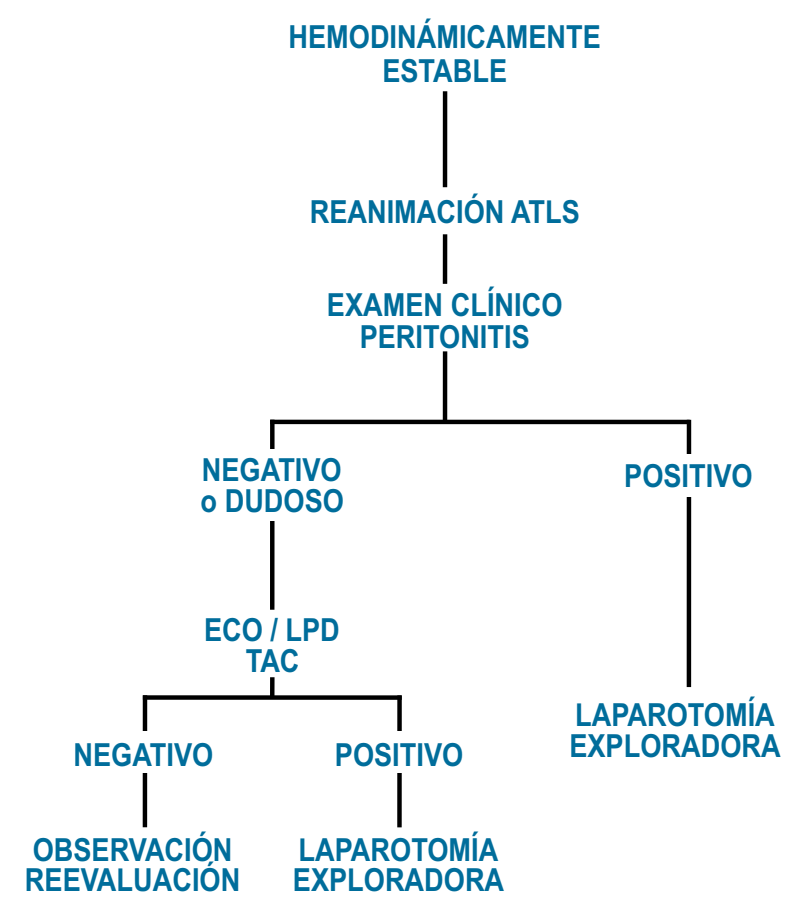

Figura 3. Trauma Abdominal Cerrado. Hemodinámicamente estable.

Cuando el paciente con trauma abdominal cerrado se encuentra hemodinámicamente inestable se realiza la reanimación y si hay signos abdominales de irritación peritoneal, distensión y dolor, se realiza exploración quirúrgica sin excepción. En caso que el examen de abdomen sea dudoso se realiza ecografía o LPD. Si es positivo se realiza laparotomía y si es negativo se deben buscar otras lesiones que justifique la inestabilidad hemodinamica entre ellas hematoma retroperitoneal o lesiones extraabdominales ${ }^{(2,7,8,15,18)}$. (Figura 4). 


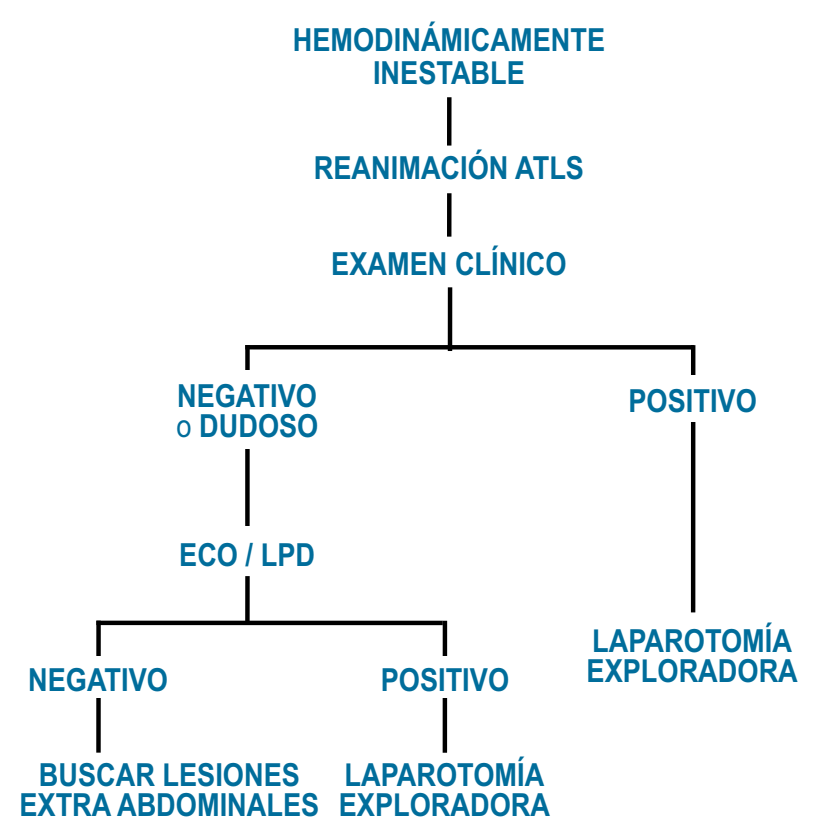

Figura 4. Trauma Abdominal Cerrado. Hemodinámicamente inestable.

\section{Trauma penetrante:}

En un paciente hemodinámicamente inestable que presenta evisceración, irritación peritoneal o sangrado del tracto gastrointestinal deberá ser llevado a sala de operaciones previa reanimación según el ATLS ${ }^{(2)}$.

En pacientes con trauma penetrante por arma blanca y hemodinámicamente estable se debe evaluar si presenta signos de irritación peritoneal. En caso de ser positivo debe realizarse una exploración quirúrgica. En caso contrario se debe explorar la herida y descartar penetración en cavidad peritoneal. De existir penetración peritoneal, en un paciente hemodinámicamente estable, se puede observar por 24 horas en controles seriados de cada 6 horas y con estudios de TAC. Si se presentara signos de irritación peritoneal se indica la laparotomía exploradora. De continuar hemodinámicamente estable y sin signos abdominales puede ser dado de alta ${ }^{(7,8,15,23,24,25)}$. (Figura 5).

En todos los pacientes con trauma penetrante en el abdomen por PAF debe realizarse una exploración quirúrgica de la cavidad abdominal. Esta regla tiene algunas excepciones: es el caso cuando la bala tiene una trayectoria tangencial a la cavidad peritoneal lo que ocurre usualmente cuando el proyectil ingresa por los flancos y no perfora el peritoneo. Igualmente, algunas escuelas consideran que las lesiones de la región toracoabdominal son tributarias de manejo conservador siempre y cuando no exista inestabilidad hemodinámica ni signos de irritación peritoneal. En este grupo de pacientes la laparoscopía juega un rol importante ${ }^{(7)}$.
De la misma manera en heridas en tórax inferior, flanco y región lumbar, se debe tener presente que puede asociarse a lesión de diafragma en primer caso y de órganos retroperitoneales $^{(8,25)}$. (Figura 5).

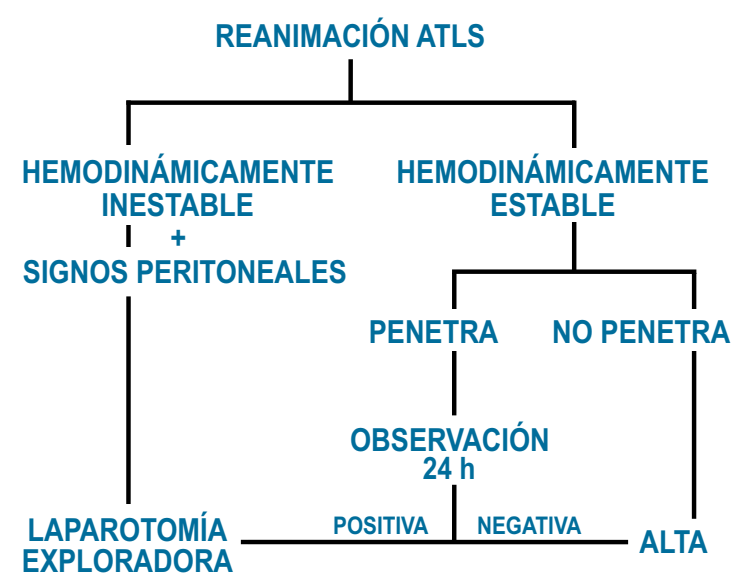

Figura 5. Trauma Abdominal Penetrante. Arma blanca.

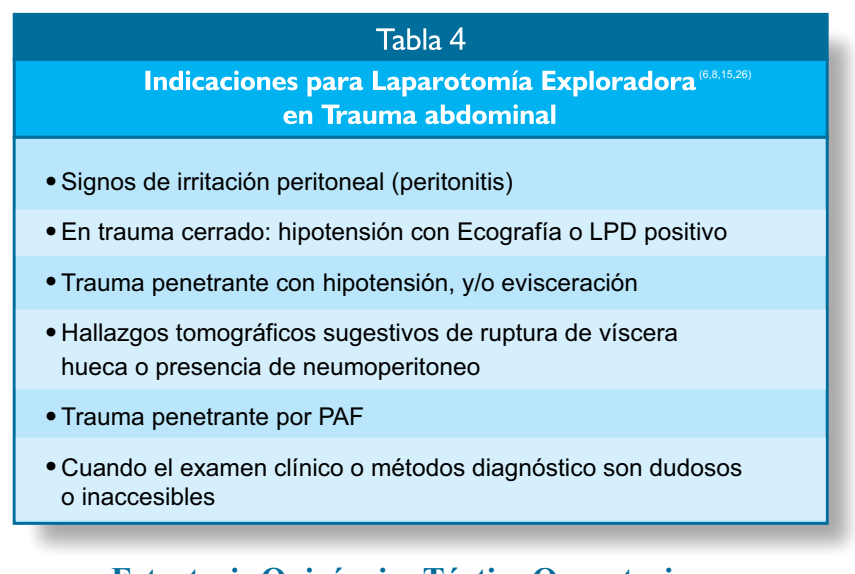

Estrategia Quirúrgica Táctica Operatoria

En los casos de trauma abdominal las lesiones pueden ser múltiples lo que obliga a realizar una exploración detallada para tener la seguridad de no pasar por alto algunas lesiones que pueden tener consecuencia en la evolución del paciente, las denominadas lesiones inadvertidas.

Un problema en las laparotomías exploradoras por trauma es la alta tasa de laparotomías innecesarias, especialmente en trauma penetrante por arma blanca, es decir aquellas intervenciones en las cuales no hay lesiones o son lesiones menores que no necesitan reparación(23-53\%) ${ }^{(27)}$. Frente a la obligación de no pasar por alto lesiones se debe considerar los siguientes puntos:

-Incisión mediana supra infraumbilical (xifopubiana)

-Aspiración de fluidos intraperitoneales: sangre, secreción intestinal 
- Coloque compresas grandes en los cuatro cuadrantes

-Identifique el origen de la hemorragia

- Descarte presencia de hematoma retroperitoneal

- Controle la hemorragia de vasos importantes

- Descarte focos de contaminación (lesión de visceras huecas)

-Examine toda la cavidad abdominal: diafragma (derecho e izquierdo), esófago intraabdominal, estómago (incluya la cara posterior), trascavidad de los epiplones, páncreas, duodeno, intestino delgado en su totalidad y su mesenterio, colon y recto intraperitoneal, vejiga y retroperitoneo.

-Si hay sospecha de lesión duodenal, se debe realizar la maniobra de Kocher

Cuando se trate de trauma penetrante por PAF se debe orientar por la probable trayectoria del proyectil ${ }^{(6,8,15)}$.

El abordaje laparoscópico se indica en pacientes hemodinámicamente estables. De preferencia Se realiza el neumoperitoneo abierto. Se utiliza cámara de 30 grados y dos forceps atraumáticos.

Se realiza una rápida evaluación del abdomen, aspiración del sangrado, coágulos y secreción intestinal. Se busca el origen de la hemorragia y cuando es posible se procede a cohibir el sangrado con pinza, electrocoagulación, ligadura o sutura. También puede utilizarse agentes hemostáticos. En caso que el sangrado no pueda ser controlado se procede a la laparotomía exploradora.

Si se controla el sangrado, se debe proceder a una revisión sistemática de toda la cavidad peritoneal, evaluando la integridad de los órganos peritoneales y retroperitoneales. Esta revisión debe realizarse siguiendo un orden preestablecido:

a. empezar con los diafragmas,

b. hígado y vesícula biliar

c. bazo

d. estómago cara anterior y posterior

e. transcavidad: páncreas y retroperitoneo supramesocólico

f. duodeno

g. intestino delgado desde el ligamento de treitz hasta la válvula ileocecal, en este paso se procede a una revisión cuidadosa y detallada de la pared intestinal y de su respectivo mesenterio, poner mayor atención en caso de hematomas en el borde mesentérico. Se recomienda realizar este examen intestinal por dos veces.

h. Colon derecho, riñón derecho y uréter i. Colon transverso

j. Colon izquierdo, riñón izquierdo y uréter

k. Cavidad pélvica: vejiga, recto y descartar presencia de hematoma retroperitoneal ${ }^{(28,29)}$.

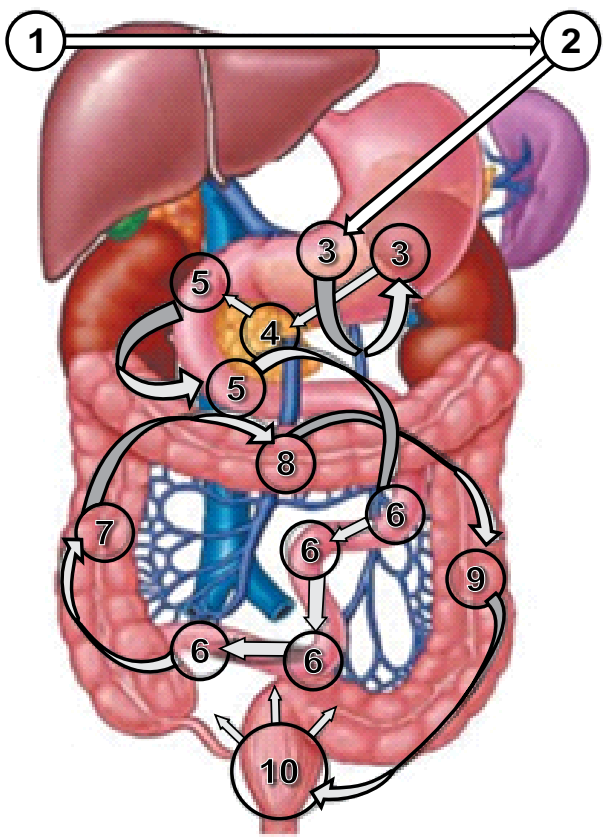

Figura 6. Exploración sistemática laparoscópica de la cavidad abdominal (Tomada de Koto 2018) $)^{(28)}$.

De acuerdo a las lesiones encontradas se realizará la reparación o resección según sea el órgano comprometido, en caso de lesión hepática las alternativas van desde una ligadura o sutura hasta el empaquetamiento. En lesión del bazo se podrá realizar esplenorrafia o esplenectomía. En lesiones de diafragma se realiza sutura asociado a drenaje torácico.

En estómago, de acuerdo a la magnitud de la lesión se puede realizar sutura simple o resección parcial.

En lesiones de intestino delgado se puede realizar la sutura simple cuando son lesiones únicas y resecciones parciales de acuerdo a la magnitud del daño.

En las lesiones que comprometan colon, la sutura simple esta indicada en lesiones de colon derecho y con menos de 8 horas de evolución. La resección y/o colostomía son alternativas de manejo en todas las otras circunstancias. En lesiones de recto, la sutura, colostomía y drenaje constituyen una buena estrategia.

Reviste particular importancia los traumatismos severos de hígado (grado III, IV y V), que necesitan de un manejo a cargo de un cirujano de experiencia, pues probablemente sea 
necesario realizar resecciones hepáticas y reparaciones vasculares. Las lesiones duodenopancreáticas son poco frecuentes, pero su manejo es altamente complejo, que puede necesitar resecciones amplias que requieren un adecuado conocimiento anatómico y experiencia ${ }^{(8)}$.

\section{Cirugía de Control de Daños}

Cuando nos enfrentamos con pacientes con trauma abdominal que involucran lesiones multiviscerales, la intervención quirúrgica demanda un tiempo prolongado para realizar la reparación de las lesiones. Es frecuente que los cirujanos intenten reparar lesiones hepáticas o realizar esplenectomía y suturar u ostomizar lesiones de vísceras huecas, que prolongan el tiempo operatorio. La mortalidad en estos casos es muy alta, $60-70 \%{ }^{(30,31)}$.

Los estudios demuestran que la causa principal de mortalidad en el intra y postoperatorio es la presencia de la tríada: acidosis, hipotermia y coagulopatía. Rotondo y col. ${ }^{(30)}$ describieron una nueva forma de enfrentar a estos pacientes a fin de disminuir la mortalidad, la denominada Cirugía de Control de Daños (Damage Control) ${ }^{(30,15)}$.

La acidosis se explica por una disminución de oxígeno por una importante pérdida sanguínea que lleva a hipovolemia e hipoperfusión celular. La presencia de hipotermia se explica por la hipovolemia, la pérdida de calor a través del peritoneo durante una laparotomía, la reanimación con líquidos a temperatura ambiental (siendo más fríos que la temperatura corporal), la transfusión sanguínea, la temperatura ambiental del quirófano, entre otros. La hipotermia produce trastorno de la cascada de la coagulación y disfunción de las plaquetas ${ }^{(30)}$.

Este procedimiento está indicado en pacientes traumatizados con shock hemorrágico profundo y secuela metabólica preoperatoria y transoperatoria la cual afecta adversamente la sobrevida. La lesión multiorgánica en la cavidad abdominal invita a realizar la Cirugía de Control de Daño (CCD). Éstas se presentan usualmente en presencia de trauma cerrado de alta energía que compromete tórax y abdomen, sospecha de trauma vascular y exsanguinación multiregional ${ }^{(31)}$.

\section{Esta fase tiene los siguientes objetivos: \\ I. Cirugía Abreviada \\ II. Manejo en UCI \\ III. Reoperación planeada}

La CCD se plantea en tres fases. En la primera fase, que es la intraoperatoria, denominada Cirugía Abreviada, se sustenta en la disminución del tiempo operatorio mediante la rápida detención del sangrado y de medidas temporales que eviten la contaminación de la cavidad peritoneal. La segunda fase se realiza en la UCI cuyo objetivo es restablecer el estado fisiológico mediante una reanimación y la tercera fase es programar al paciente para una cirugía electiva para dar solución final a los problemas ${ }^{(32)}$.

Algunos autores mencionan la fase cero, como aquella que involucra un transporte rápido, reanimación con líquidos y prevención de la hipotermia ${ }^{(31)}$.

\section{Cirugía Abreviada}

La cirugía abreviada, la primera fase, tiene como principio disminuir el tiempo quirúrgico, la idea que se cumpla con los objetivos en el menor tiempo posible. Se debe realizar la "cirugía mínima" para controlar el sangrado y la fuga de contenido intestinal o urinario. Hay que evitar procedimientos complejos o de reconstrucción que consumen tiempo y prolonguen la operación.

Durante el transoperatorio, se debe utilizar ante la presencia de:

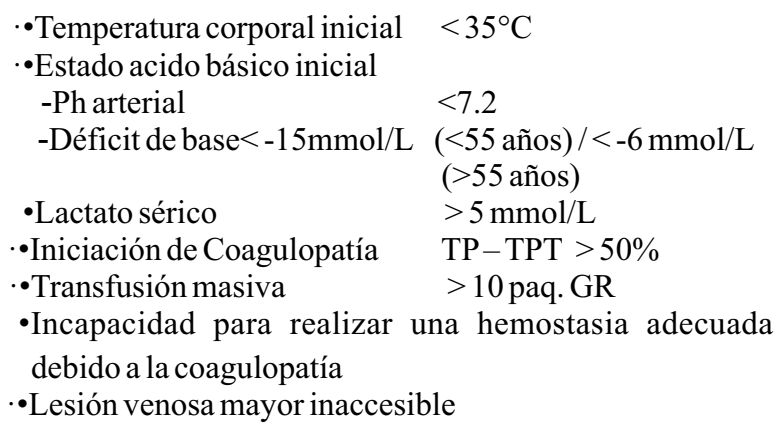

Esta fase tiene los siguientes objetivos:

A. Control de hemorragia

B. Control de contaminación

C. Cierre temporal de la pared

A. El control de la hemorragia se debe realizar como objetivo primario, una vez identificada la fuente del sangrado, se debe comprimir con compresas, evaluar los diferentes procedimientos que disponemos, desde electrocoagulación, pinzamiento y ligadura, reparación y sutura hasta empaquetamiento. La experiencia del operador deberá distinguir entre una hemorragia por coagulopatía y de origen de un vaso sanguíneo importante, en este caso el empaquetamiento no esta indicado, sino la ligadura o sutura del vaso comprometido. 
Se recomienda que el cirujano cuente con una adecuada exposición, para lo cual puede ser necesario ampliar la incisión, y de ser necesario pedir ayuda competente y además debe comunicarlo a todo el equipo quirúrgico.

Recordar que en la cavidad abdominal se puede ligar todos lo vasos a excepción de arteria mesentérica superior, arteria renal y arteria ilíaca ${ }^{(31)}$.

El empaquetamiento, también llamado "packing”, se debe utilizar en presencia de hipotermia menor o igual de 34 grados, y en una hemorragia severa secundaria a trauma hepático grave, lesión vascular o hematoma retroperitoneal expansivo. Esta decisión debe tomarse en los primeros minutos (10-15) de la intervención quirúrgica ${ }^{(30)}$.

La demora en tomar esta decisión esta asociada a una elevada mortalidad, por lo que se debe considerar como una medida terapéutica primaria ${ }^{(32)}$.

B. El control de la contaminación esta referido a evitar una diseminación de fugas del contenido intestinal, se recomienda evitar las resecciones y anastomosis primaria pues prolongan el acto operatorio, si es necesario resecar, se grapan o suturan los cabos intestinales. Tampoco se recomiendan las ostomías.

C. Finalmente se prefiere realizar un cierre temporal de la pared, recordar que este paciente deberá ser reintervenido a las 48-72 horas. No se debe realizar el cierre formal de la pared, es decir por planos, consume tiempo y puede originar hipertensión intraabdominal. Se utiliza la denominada Bolsa de Bogotá (actualmente hay dispositivos con tecnología que lo hacen más efectiva), que se sutura a la piel, con una sutura continua, lo que es rápido y efectivo. Se puede utilizar bolsas de plástico previamente esterilizadas, nosotros utilizamos las bolsas colectoras de orina. Este cierre previene la presentación del Síndrome Compartamental y nos permite observar el tipo de secreciones en la cavidad peritoneal ${ }^{(30,33,34,31,8)}$.

Si bien no existe un tiempo establecido, se recomienda que el acto operatorio no debe durar más de 120 - 150 minutos. (32).

\section{Manejo en UCI}

Luego de la intervención quirúrgica, el paciente idealmente debería pasar a la Unidad de Cuidados Intensivos, donde el objetivo principal será revertir la hipotermia, coagulopatía y acidosis. El paciente recibe todos los líquidos recalentados, se maximixan los parámetros hemodinámicos con un adecuado soporte ventilatorio y se corrige la acidosis.
Las primeras 24 horas son las importantes en este tipo de pacientes, una vez controlada la triada de hipotermia, coagulopatía y acidosis, se procede a la tercera fase $\mathrm{f}^{(7,8,30)}$.

\section{Reoperación planeada}

A pesar que no hay un tiempo preciso para realizar esta fase, se considera de 48 a 72 horas un tiempo adecuado, sobretodo cuando existe empaquetamiento en el procedimiento. Algunos autores prefieren el tiempo de 36 horas cuando existen asas ciegas por ligadura. En esta fase se realiza la extracción del empaquetamiento, que no siempre es un procedimiento sencillo por lo que deberá proceder con el cuidado necesario $^{(7)}$.

Durante la intervención quirúrgica se debe hacer un lavado de la cavidad con solución salina y una exploración de toda la cavidad para identificar lesiones inadvertidas.

El tiempo operatorio no es fundamental pues la triada de acidosis, hipotermia y coagulopatía fue superada en la UCI, por lo tanto podemos realizar todas las maniobras de reparación y reconstrucción necesarias ${ }^{(30)}$.

Cuando al retirar el empaquetamiento, el sangrado persiste y no cede a otras maniobras, está indicado el reempaquetamiento $^{(32)}$.

Con la implantación de la CCD se ha logrado que la supervivencia sea de $58 \%$, es decir la mortalidad se ha reducido casi a la mitad ${ }^{(7,8,15,30,34)}$. (Figura 7).

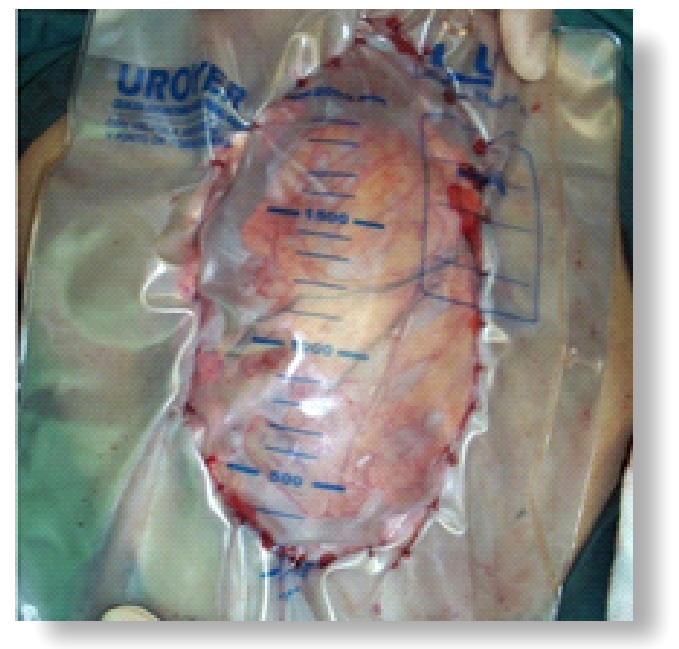

Figura 7. Bolsa de Bogotá utilizando bolsa colectora de orina.

El manejo del trauma abdominal es complejo y requiere la participación de otros especialistas, cirujanos, emergencistas, anestesiólogos, intensivistas, imagenólogos, entre otros, 
con las competencias que le permitan un desempeño óptimo en el diagnóstico y tratamiento de los paciente que generalmente son jóvenes y con expectativas de vida que no pueden ser truncadas por falta de entrenamiento del equipo médico.

\section{Referencias bibliográficas}

1. González G. Epidemiología del trauma. En Morales $\mathrm{CH}$, Isaza L, editors. Carlos Morales, Luis Isaza. Cirugía Trauma Primera ed. Medellin: Universidad de Antioquía; 2004:3-12.

2. American College of Surgeons. ATLS Soporte Vital Avanzado en trauma. Novena ed. American College of Surgeons. Chicago: ACS; 2012.

3. Ospina J, Chica C, Cabal A. Índices de severidad del trauma y la enfermedad. En Carlos Ordoñez, Ricardo Ferrada, Ricardo Buitrago. Cuidado Intensivo y Trauma. Bogotá: Distribuna; 2002:383-402.

4. American Association Surgeons of Trauma. [Internet] Injury Scoring Scala https://www.aast.org/library/traumatools/ injuryscoringscales.aspx

5. Maurer A, Morris J. Injury Severity Scoring. En MooreFeliciano-Mattox. Trauma. New York: McGraw-Hill; 2004: 87-91.

6. Sánchez R. Lama T, Carrillo E. Trauma Abdominal. En Ricardo Ferrada, Aurelio Rodríguez. Trauma Sociedad Panamericana de Trauma. Bogotá: Distribuna; 2009:307-315.

7. Henao C. Trauma Abdominal. En Carlos Morales, Luis Isaza . Cirugía Trauma. Medellin: Universidad de Antioquía; 2004:387-402.

8. Simone A, Frankel H, Velmahos G. Abdominal Injury. En Peitzman A, Rhodes M, Schwab C, Yealy D, Fabian T. The Trauma Manual. Philadelphia: Lippincott Williams Wilkins; 2008:243-282.

9. Sakran JV, Ezzeddine H, Schwab CW, Bonne S, Brasel KJ, Burd RS, Cuschieri J, Ficke J, Gaines BA, Giacino JT, Gibran NS, Haider A, Hall EC, Herrera-Escobar JP, Joseph B, Kao L, Kurowski BG, Livingston D, Mandell SP, Nehra D, Sarani B, Seamon M, Yonclas P, Zarzaur B, Stewart R, Bulger E, Nathens. Proceedings from the Consensus Conference on Trauma Patient-Reported Outcome Measures. J Am Coll Surg. 2020;230(5):819-835.

10. MINSA Centro Nacional de Epidemiología, Prevención y Control de Enfermedades. [Internet] Lima. Situación de las lesiones causadas por accidentes de transito en el Perú $2^{\circ}$ Trimestre 2019; 2019. Disponible en: https://www.dge.gob.pe/ portal/docs/vigilancia/sala/2019/SE24/transito.pdf

11. Demetriades D, Inabe K. Abdominal Injury. En Demetrios Demetriades, Edward J. Newton. Emergency Trauma. New York: Cambridge University; 2011:122-141.

12. Romero N. Características del Trauma Abdominal Abierto Hospital Nacional Sergio E. Bernales 2016 [Tesis para optar el Título de Especialista] Facultad de Medicina UPSMP 2018

13. Astudillo R. Actualización en Trauma. Primera ed. Cuenca: Laboratorios Interpharm; 2004.

14. Group PGU. Level 3 guideline on the treatment of patients with evere/multiple injuries: AWMF Register-Nr. 012/019. Eur. J. Trauma. Emerg. Surg. 2018(Suppl 1);44(3):3-271.

15. Rodriguez F, Viteri Y. Trauma Abdominal. En Rodriguez F.
Viteri Y, Vivas L,Ottolino P. Manejo del Paciente Traumatizado. Caracas: Básica 1;2003. p. 291-308.

16. Broos, P LO, Gutermann H. Actual Diagnostic Strategies in Blunt Abdominal Trauma. European Journal of Trauma, 2002;28(2):64-74.

17. Pacheco AM. Trauma Abdominal. Rev. Med. Clin. Condes. 2011;22(5):623-630.

18. Stengel D, Rademacher G, Ekkernkamp A, Güthoff C, Mutze S. Emergency ultrasound-based algorithms for diagnosing blunt abdominal trauma. Cochrane Database Syst Rev. 2015;14;2015(9):CD004446.

19. Holmes JF, Harris D, Battistella FD. Performance of abdominal ultrasonography in blunt trauma patients with outof-hospsital or emergency department hypotension. Ann Emerg Med. 2004;43(3):354-361.

20. O'Malley E, Boyle E, O'Callaghan A, Coffey JC, Walsh SR. Role of laparoscopy in penetrating abdominal trauma: a systematic review. World J Surg. 2013;37(1):113-122.

21. Ivatury R. Montbrun E. Laparoscopía en Trauma. En Ricardo Ferrada, Aurelio Rodríguez. Trauma Sociedad Panamericana de Trauma. Bogotá: Distribuna; 2009:131-136.

22. Hanna W. Fata P, Ferri L, Razek T, Ortega D. Trauma de Diafragma. En Ricardo Ferrada, Aurelio Rodríguez. Trauma Sociedad Panamericana de Trauma. Bogotá: Distribuna; 2009: 329-336.

23. Biffl WL, Leppaniemi A. Management guidelines for penetrating abdominal trauma World J Surg 2015;39(6):13731380.

24. Butt MU, Zacharias N, Velmahos GC. Penetrating abdominal injuries: management controversies. Scand J Trauma Resusc Emerg Med. 2009; 17:19.

25. Gonzáles A GA. Trauma Abdominal Penetrante. En Ricardo Ferrada, Aurelio Rodríguez. Trauma Sociedad Panamericana de Trauma. Bogotá: Distribuna; 2009:317-328.

26. Demetriades D, Velmahos G. Indications for laparotomy. En Feliciano DV, Moore E, Mattox K. Trauma. 5th ed.: Mc Graw Hill; 2004:593-612.

27. Como JJ, Bokhari F, Chiu WC, Duane TM, Holevar MR, Tandoh MA, Scalea TM. Practice Management Guidelines for Selective Nonoperative Management of Penetrating Abdominal Trauma. The Journal of Trauma: Injury, Infection, and Critical Care. 2010;68(3):721-733.

28. Koto MZ, Matsevych OY, Aldous C. Diagnostic Laparoscopy for Trauma: How not miss injuries J Laparoendosc Adv Surg Tech 2018;28(5):506-513.

29. Kawahara NT, Alster C, Ujimura RS, Birolini D. Standard examination system for laparoscopy in penetrating abdominal trauma J Trauma 2009;67(3):589-595.

30. Carlos Ordoñez. Control de Daños. En Ordoñez C, Ferrada R, Buitrago R. Cuidado Intensivo y Trauma. Bogotá : Distribuna; 2002:429-450. 
31. Schwab C, Liao A, RotondoM, Soto R. Control de Daños. En Ricardo Ferrada, Aurelio Rodríguez. Trauma Sociedad Panamericana de Trauma. Bogotá: Distribuna; 2009:535-542.

32. Toschlog E, Sagraves S, Rotondo M. En Peitzman A, Rhodes M, Schwab C, Yealy D, Fabian T. The Trauma Manual. Philadelphia: Lippincott Williams Wilkins; 2008:283-292.

33. Carlos Ordoñez, Luis Ramos. Hipertensión intraabdominal y Síndrome de Compartmiento abdominal. En Ordoñez C,
Ferrada R, Buitrago R. Cuidado Intensivo y Trauma. Bogotá : Distribuna; 2002: 539-555.

34. Rotondo MF, Schwab CW, McGonigal MD, Phillips GR 3rd, Fruchterman TM, Kauder DR, Latenser BA, Angood PA. "Damage Control": an approach for improved survival in exsanguinating penetrating abdominal injury. J Trauma. 1993 ; $35(3): 375-382$.

Contribución de autoría: David A. Ortega-Checa ha participado en la concepción del artículo, la redacción del manuscrito, la revisión crítica del manuscrito y aprobación de su versión final. Iván Vojvodic-Hernández ha participado en la concepción del artículo, la redacción del manuscrito, la revisión crítica del manuscrito y aprobación de su versión final.

Conflicto de interés: Los autores no tienen conflicto de interés con la publicación de este trabajo.

Financiamiento: Autofinanciado.

Citar como: Ortega-Checa DAy col.. Trauma Abdominal. Diagnóstico(Lima). 2021;60(1):23-34

DOI: 10.33734/diagnostico.v60i1.274

Correspondencia: David A. Ortega Checa. Correo electrónico: davidortegach@gmail.com

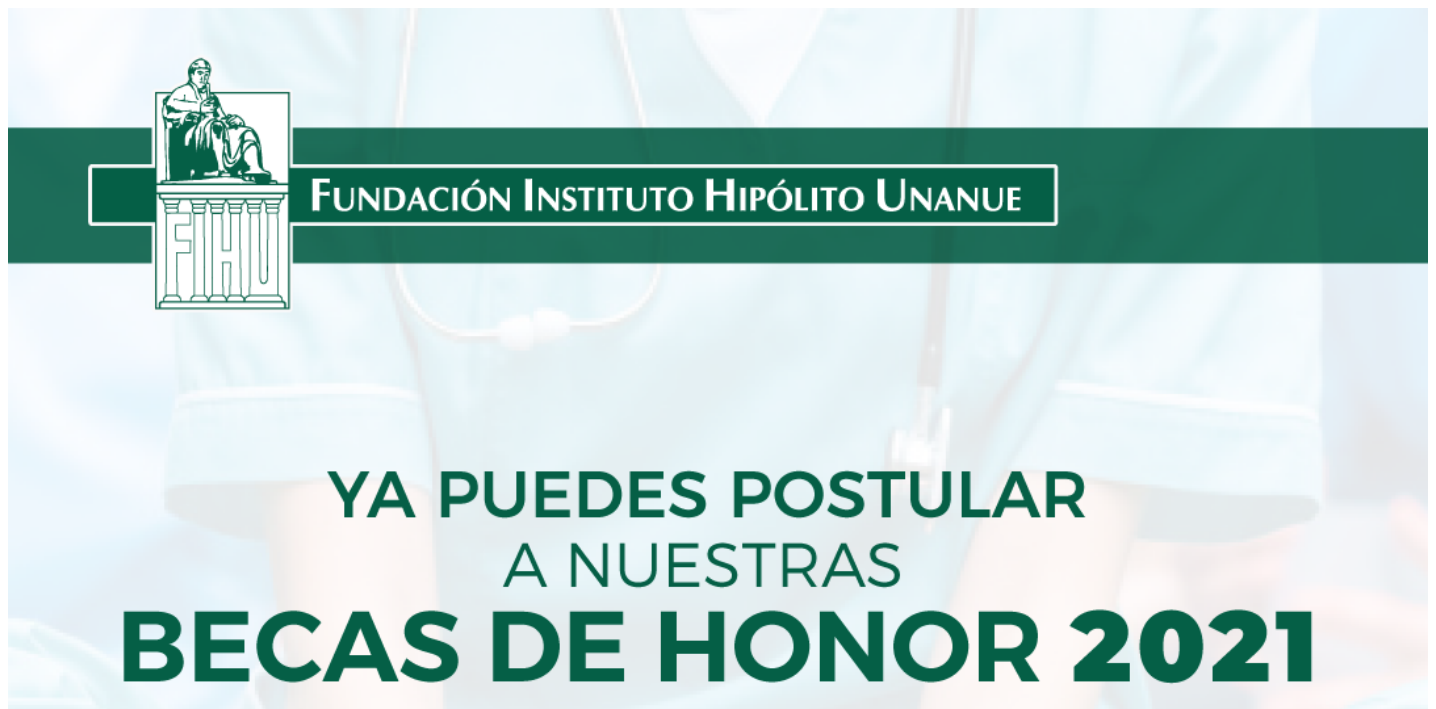

Envía tu postulación al siguiente correo:

fihu-diagnostico@alafarpe.org.pe

La fecha límite es $\mathbf{3 0}$ de abril 